Open Access

\title{
Chronic inflammatory demyelinating polyneuropathy (CIDP): change of serum IgG dimer levels during treatment with intravenous immunoglobulins
}

\author{
Christian Ritter ${ }^{1,2,3}$, llja Bobylev ${ }^{1,2}$ and Helmar C. Lehmann ${ }^{1,2^{*}}$
}

\begin{abstract}
Background: Intravenous immunoglobulin (IVIg) is an effective treatment in chronic inflammatory demyelinating polyneuropathy (CIDP). In most patients, the optimal IVlg dose and regime is unknown. Polyvalent immunoglobulin (Ig) G form idiotypic/anti-idiotypic antibody pairs in serum and IVlg preparations. We determined lgG dimer levels before and after IVIg treatment in CIDP patients with the aim to explore their utility to serve as a surrogate marker for treatment response.

Methods: IgG was purified from serum of five controls without treatment, as well as from serum of 16 CIDP patients, two patients with Miller Fisher syndrome (MFS), and one patient with myasthenia gravis before and after treatment with IVlg. IgG dimer levels were determined by size exclusion chromatography. IgG dimer formation was correlated with clinical response to IVlg treatment in CIDP. Re-monomerized IgG dimer fractions were analyzed for immunoreactivity against peripheral nerve tissue.

Results: IgG dimer levels were significantly higher in post- compared to pre-IVlg infusion samples. Low post-treatment IgG dimer levels in CIDP patients were associated with clinical worsening during IVIg treatment. Re-monomerized lgG dimer fractions from CIDP patients showed immunoreactivity against peripheral nerve tissue, whereas similarly treated samples from MFS patients showed immunoreactivity against GQ1b.

Conclusion: Assessment of IgG dimer levels could be a novel approach to monitor CIDP patients during IVIg treatment, but further studies in larger cohorts are warranted to explore their utility to serve as a potential therapeutic biomarker for IVIg treatment response in CIDP.
\end{abstract}

Keywords: Immune neuropathy, Surrogate marker, Inflammation, Autoantibody

\section{Background}

Chronic inflammatory demyelinating polyneuropathy (CIDP) belongs to the most common immune-mediated chronic disorders of the peripheral nervous system. Abundant experimental evidence suggests that CIDP is caused by an aberrant immune response that involves autoreactive $\mathrm{T}$ cells, macrophages, and autoantibodies [1-4]. However, despite extensive research over the last

\footnotetext{
* Correspondence: helmar.lehmann@uk-koeln.de

'Department of Neurology, University Hospital Cologne, Cologne, Germany ${ }^{2}$ Center of Molecular Medicine Cologne (CMMC), University of Cologne, Cologne, Germany

Full list of author information is available at the end of the article
}

decades, the target antigens of the humoral and cellmediated immune response are yet poorly defined.

Intravenous immunoglobulin (IVIg) is considered the first-line treatment for CIDP, and several controlled studies demonstrated its short- and long-term efficacy [5-7]. The therapeutic effect of IVIg in CIDP is not fully understood, but it is believed that different modes of action are involved. These mechanisms include blockage or modulation of Fc $\gamma$-receptors, regulation of $\mathrm{T}$ cell and $B$ cell activation, and alteration of inflammatory cytokines [8-10]. In addition, there is evidence from other autoimmune diseases that IVIg contains anti-idiotypic (anti-Id) antibodies that are able to neutralize pathological 
idiotypic autoantibodies (Id) by forming Id-anti-Id complexes [11-15].

IVIg preparations contain pooled IgG from different donors and are mostly monomeric IgG. However, a small percentage of polyvalent IgG form Id-anti-Id antibody dimers [16, 17]. Increased levels of IgG dimers have been linked to adverse events during IVIg infusion [18]. In contrast, there are experimental studies that suggest beneficial effects of increased IgG dimer levels in IVIg preparations [19].

Dimeric IgG is also detectable in the serum of healthy individuals, although its amount compared to monomeric IgG is usually much lower than that in IVIg preparations [20, 21]. Notably, it is currently unknown if IVIg treatment influences the circulating IgG dimer content in humans with immune-mediated neurological diseases.

We hypothesized that in CIDP, IVIg treatment may result in the formation of new Id-anti-Id complexes that could be assessed by quantification of Ig dimers in posttreatment serum of CIDP patients. We anticipated that correlations between IgG dimer formation and clinical response in individual patients could serve as a potential surrogate marker for treatment response in CIDP, in which optimal dose and regimen of IVIg is still unknown. Moreover, purified IgG dimers, containing Id-anti-Id complexes, could be employed as a novel approach to identify idiotypic (auto)antibodies and their targeted antigens in CIDP.

\section{Methods \\ Patients}

Sixteen patients with CIDP (mean age $64.4 \pm 12.2$, nine males, seven females) were included in the study. All patients were diagnosed according to diagnostic criteria developed by the Peripheral Nerve Society [22]. Fourteen of 16 patients were on maintenance therapy with IVIg and received a standard dose of $1 \mathrm{~g} / \mathrm{kg}$ bodyweight (BW). Blood was obtained immediately before and 30 min after IVIg infusion. All patients underwent a detailed clinical workup prior to the IVIg infusion, which also included assessment of the Medical Research Council (MRC) sum score and Inflammatory Neuropathy Cause and Treatment (INCAT) disability score as previously described [23] (Table 1). Patients were considered stable when INCAT score and MRC sum score remained unchanged over 6 months of IVIg treatment, and worsening to IVIg treatment was defined as change of either INCAT or MRC sum score within 6 months.

In addition, blood was obtained from three patients suffering from other immune-mediated neurological diseases (myasthenia gravis, MG, $n=1$; Miller Fisher syndrome, MFS, $n=2$ ) immediately before and $30 \mathrm{~min}$ after IVIg infusion and from five control patients (mean age
$51.8 \pm 13.9$, two females, three males). These included patients with neurological diseases other than CIDP, MG, or MFS (idiopathic cephalgia, peripheral facial nerve paralysis $(n=2)$, subarachnoid hemorrhage, epilepsy).

The study is registered with ClinicalTrials.gov, number NCT01655394, and approved by the local ethics committee (Ethics Committee University of Cologne, \#12-182). All patients gave written informed consent prior to inclusion into the study.

\section{Chromatographic separation of monomeric and dimeric IgG fractions}

Blood samples of patients were centrifuged $(2000 g$, $15 \mathrm{~min}$ ), and serum was prior to preparation of IgG fractions diluted 1:4 with low-salt buffer (LS buffer) containing $100 \mathrm{mM}$ HEPES and $10 \mathrm{mM} \mathrm{NaCl}$ at $\mathrm{pH}$ 7.5. All IgG purification experiments were performed on an automated fast protein liquid chromatography system (AKTA, GE Healthcare Bio-Sciences Corp.) using Unicorn software (GE Healthcare Bio-Sciences Corp.) for analysis.

First, IgG fractions were purified from CIDP serum samples using ion exchange chromatography columns (HighTrap Q HP and HighTrap SP HP, GE Healthcare). Columns were calibrated using LS buffer, and serum samples were injected with a $1 \mathrm{ml}$ sample loop. IgG fractions were dissolved from the HighTrap SP HP column using high-salt (HS) final buffer (100 mM HEPES and $500 \mathrm{mM} \mathrm{NaCl}$ at $\mathrm{pH}$ 7.5). A gradient was generated over $20 \mathrm{~min}$ at a defined flow rate. Collected IgG fractions were re-concentrated to a $1 \mathrm{ml}$ volume using a commercial protein concentration kit (Pierce) according to the manufacturer's protocol.

The molecular weight (MW) profile of dimeric and monomeric IgG was determined by gel filtration chromatography using an analytical-grade column (Superdex 200, GE Healthcare) and standard gel filtration buffer at a flow rate of $0.5 \mathrm{ml} / \mathrm{min}$.

\section{SDS-PAGE analysis of monomeric and dimeric lgG fractions}

The purity of dimeric and monomeric IgG fractions was checked by sodium dodecyl sulfate-polyacrylamide gel electrophoresis (SDS-PAGE) using nUVview precast Tris-HEPES gels containing $10 \%$ polyacrylamide. Bands were visualized under ultraviolet (UV) light after a 2-min exposure time. As a standard, the low molecular weight $(\mathrm{LMW})$ references from Bio-Rad were used.

\section{Detection of antibodies against GQ1b and AchR}

Commercial ELISA plates (Sigma-Aldrich) were coated with human GQ1b (Sigma-Aldrich). GQ1b-coated microtiter plates were blocked with $1 \%$ BSA solution for $2 \mathrm{~h}$ at $4{ }^{\circ} \mathrm{C}$ and removed by washing with phosphatebuffered saline (PBS) afterwards. Dimer IgG fraction post-IVIg treatment was re-monomerized by dialyzing 
Table 1 Clinical characteristics from CIDP patients with INCAT disability score, MRC sum score, and treatment history

\begin{tabular}{|c|c|c|c|c|c|c|c|c|c|c|}
\hline Patient & Sex & Age & $\begin{array}{l}\text { Treatment before } \\
\text { sampling }\end{array}$ & $\begin{array}{l}\text { IVlg treatment duration } \\
\text { before } \mathrm{d} 1 \text { (months) }\end{array}$ & $\begin{array}{l}\text { INCAT } \\
\text { score d1 }\end{array}$ & $\begin{array}{l}\text { INCAT score } \\
6 \text { months }\end{array}$ & $\begin{array}{l}\text { MRC } \\
\text { SS d1 }\end{array}$ & $\begin{array}{l}\text { MRC SS } \\
6 \text { months } \\
\end{array}$ & $\begin{array}{l}\text { Dimer content } \\
\text { pre-IVlg d1 (\%) }\end{array}$ & $\begin{array}{l}\text { Dimer content } \\
\text { post-IVIg d1 (\%) }\end{array}$ \\
\hline 1 & $M$ & 58 & CS & 0 & 2 & 2 & 58 & 58 & 1.3 & 3.0 \\
\hline 2 & $M$ & 62 & CS, IVlg & 9 & 2 & 2 & 58 & 58 & 3.8 & 11.3 \\
\hline 3 & M & 67 & CS, IVlg & 16 & 1 & 2 & 60 & 60 & 5.4 & 5.5 \\
\hline 4 & $M$ & 34 & CS, IVlg & 10 & 1 & 2 & 52 & 52 & 2.4 & 2.4 \\
\hline 5 & $\mathrm{~F}$ & 79 & CS, IVlg & 28 & 7 & 6 & 52 & 52 & 4.6 & 9.3 \\
\hline 6 & M & 61 & IVlg & 16 & 2 & 2 & 58 & 58 & 1.8 & 4.2 \\
\hline 7 & $M$ & 74 & CS, IVlg & 27 & 7 & 7 & 46 & 46 & 3.7 & 11.3 \\
\hline 8 & $F$ & 69 & IVlg & 5 & 0 & 0 & 60 & 60 & 2.7 & 6.0 \\
\hline 9 & $F$ & 73 & $C S, C P$ & 6 & 4 & 7 & 52 & 47 & 2.3 & 4.1 \\
\hline 10 & M & 48 & CS, IVlg & 6 & 3 & 1 & 58 & 58 & 2.1 & 3.4 \\
\hline 11 & $F$ & 56 & IVlg & 2 & 3 & 3 & 58 & 58 & 2.1 & 2.3 \\
\hline 12 & $F$ & 71 & IVlg & 8 & 0 & 0 & 60 & 60 & 3.9 & 7.4 \\
\hline 13 & $F$ & 79 & IVlg & 10 & 2 & 2 & 52 & 52 & 1,9 & 7,7 \\
\hline 14 & M & 65 & IVlg & 10 & 2 & 2 & 58 & 58 & 0 & 4.2 \\
\hline 15 & $F$ & 78 & CS, IVlg & 12 & 1 & 1 & 58 & 58 & 3.7 & 6.2 \\
\hline 16 & $M$ & 57 & - & 0 & 2 & 2 & 60 & 60 & 0 & 3.9 \\
\hline
\end{tabular}

CS cortisone, CP cyclophosphamide, SS sum score

against $10 \mathrm{mM}$ acetic acid at $\mathrm{pH} 4.0$ for $24 \mathrm{~h}$. Pre-IVIg treatment monomeric IgG and post-IVIg treatment monomeric and dimeric IgG fractions were added and incubated overnight at $4{ }^{\circ} \mathrm{C}$.

After washing with TWEEN-20 washing buffer, peroxidase-conjugated anti-human IgG was added and incubated for $2 \mathrm{~h}$ at $4{ }^{\circ} \mathrm{C}$. After another washing step, OPD solution (Sigma-Aldrich) was added according to the manufacturer's instructions for $30 \mathrm{~min}$ at room temperature (RT). Finally, the stop solution was added and optical density (OD) was measured within $30 \mathrm{~min}$ at $492 \mathrm{~nm}$. Measurements were performed in triplets. Acetylcholine receptor antibodies in pre- and posttreatment samples were determined by standard radio immune precipitation assay.

\section{Immunohistochemistry using monomeric lgG}

Dimeric IgG fractions were dialyzed against $10 \mathrm{mM}$ acetic acid, $\mathrm{pH} 4.0$, at $4{ }^{\circ} \mathrm{C}$ for $24 \mathrm{~h}$ and stored at $-20{ }^{\circ} \mathrm{C}$ until further use.

Ten-micrometer sections from frozen rat sciatic nerve samples were cut on a cryostat and collected on Superfrost ${ }^{\circ}$ Plus glass slides (Thermo Scientific). Following incubation at $-80^{\circ}$ C overnight, sections were thawed at RT for $1 \mathrm{~h}$ in a dry chamber. After 1-h incubation in blocking solution (PBS containing $10 \%$ normal goat serum and $0.4 \%$ Triton X-100), sections were incubated overnight in a humidified chamber at RT with antibodies and developed with appropriate secondary antibodies. The following antibodies were used: monoclonal mouse anti-myelin basic protein (Abcam, 1:500) and monoclonal mouse anti-beta-III tubulin (Abcam, 1:500). For immunofluorescence, we used fluorescein antibody (horse anti-mouse IgG, 1:200; goat anti-human IgG). Sections were co-stained with Hoechst 33342 (Thermo Scientific) and mounted with Fluoromount- $\mathrm{G}^{\mathrm{ma}}$ (SouthernBiotech).

\section{Statistical analysis}

Differences between serum samples and cell samples before and after treatment were analyzed by $t$ test (two groups), and data were checked for normality using the KolmogorovSmirnov test. A $p$ value $<0.05$ was considered statistically significant. Data is presented as mean $+/$ - standard deviation.

\section{Results}

Analysis of IgG dimer content before and after treatment with IVIg

We first analyzed the dimer content in serum IgG fractions before (pre) and after (post) IVIg infusion in patients with CIDP, MG, and MFS. Dimeric IgG fractions could be detected in most serum samples and varied between 0 and $11.3 \%$ of total IgG (Fig. 1a, b). In CIDP patients, there was no association between IgG dimer levels pre- and post-IVIg treatment and age, body weight, or disease severity. IgG dimer levels were significantly higher in post- compared to pre-infusion samples (dimer content pre-IVIg $2.6 \% \pm 1.5$, post-IVIg $5.8 \% \pm 2.9$, Fig. 1c). We also measured the percentage of IgG dimers in five controls and found similar IgG dimer levels compared to CIDP patients (dimer content $2.4 \pm 1.3 \%$ ). Further, we assessed the dimer content in different lots of two 


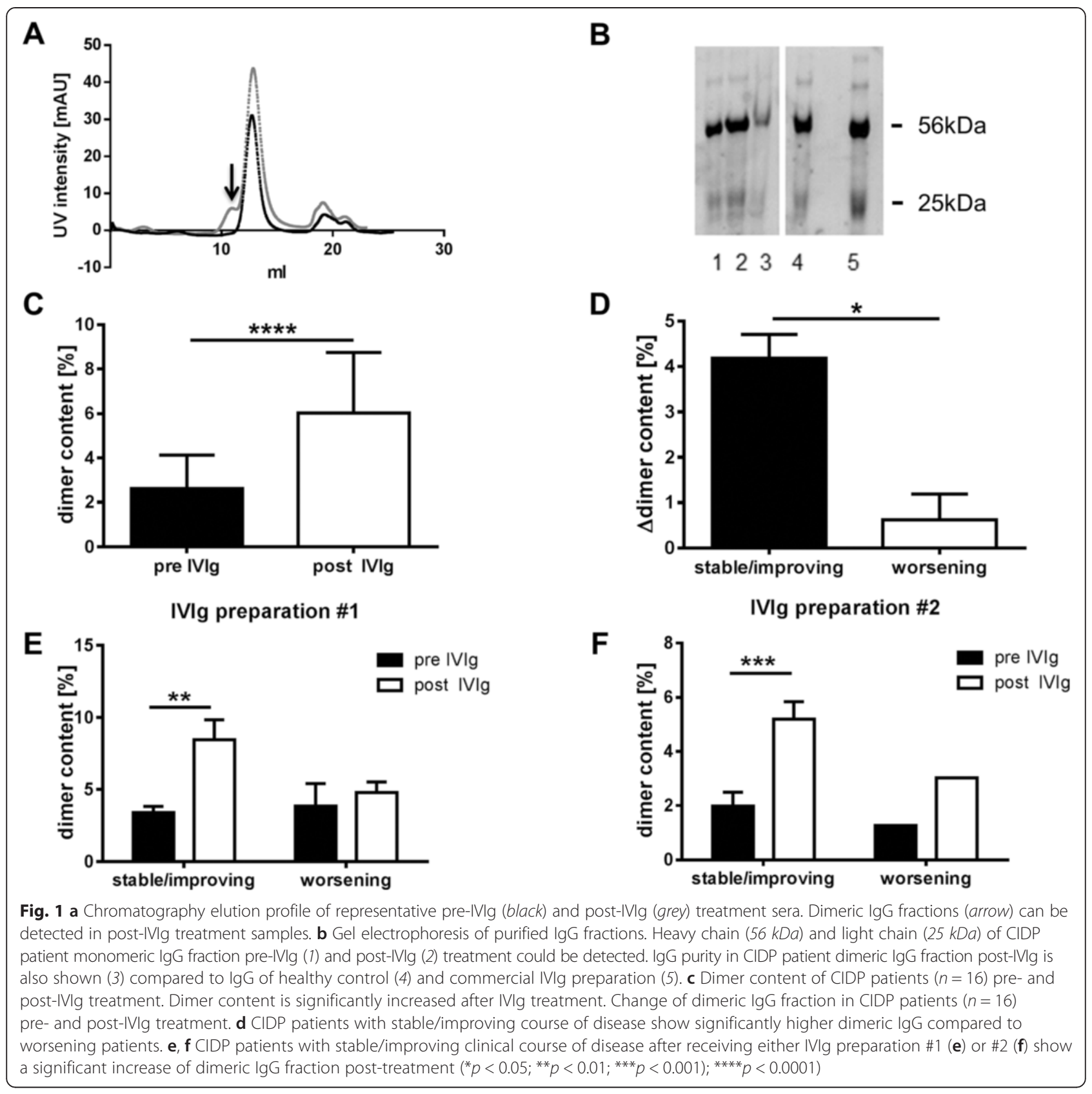

commercial IVIg preparations (Privigen ${ }^{\oplus}$, CSL Behring and Gamunex ${ }^{\oplus}$, Grifols) that were used in our patients and found higher values $(4.0 \% \pm 2.3$ and $4.9 \% \pm 1.5$, respectively) compared to the average percentage in healthy controls and pre-treatment serum samples from CIDP patients.

High IgG dimer levels after IVIg treatment are associated with disease stabilization

To assess the utility of IgG dimer levels as a surrogate marker for treatment response to IVIg, we grouped our cohort of CIDP patients according to their clinical response to IVIg treatment (stable/improving vs. worsening) and calculated the change of IgG dimer values in those two groups. CIDP patients who showed improvement or stabilization of the disease course during IVIg treatment $(n=13)$ had significantly elevated post-IgG dimer levels compared to those who worsened under IVIg treatment $(n=3$, Fig. 1d). This difference could be detected irrespective which of the two available IVIg preparations were used (Fig. 1e, f). 


\section{Autoantibody testing in newly formed dimeric IgG after IVIg treatment}

There is strong experimental evidence that IgG dimers, which are detectable in pooled human IgG fractions from different individuals, consist of Id-antiId antibody pairs. Therefore, it is likely that in CIDP, post-treatment IgG dimers may also contain Id-autoantibodies and their anti-idiotypes. This hypothesis cannot be directly validated in CIDP, since the target antigens of the presumed autoantibodies in CIDP are unknown.

To further characterize the nature of IgG dimers that emerge after IVIg infusion, we collected monomeric and dimeric IgG peaks from two patients with MFS and from one patient with MG who were treated with IVIg. Patients \#1 and \#2 were seropositive for anti-GQ1b IgG antibodies, and patient \#3 had serum IgG antibodies against acetylcholine receptors $(71 \mathrm{nmol} / \mathrm{l})$. We anticipated that IVIg dimers, which occur after IVIg treatment as a result of Id-anti-Id binding, should contain measurable amounts of those autoantibodies. Therefore, we remonomerized the dimer fraction by dialyzing against $10 \mathrm{mM}$ acetic acid at $\mathrm{pH} 4.0$ and tested pre-IVIg treatment monomeric IgG and post-IVIg treatment monomeric and dimeric IgG fractions for immune reactivity against GQ1b and AchR, respectively. Monomeric and dimeric IgG fractions of the patient with myasthenia displayed reactivity against AchR (pre-monomeric $969 \pm 10.1 \mathrm{cpm}$, post-dimeric $127 \pm 6.5 \mathrm{cpm}$, post-monomeric $547.2 \pm 16 \mathrm{cpm})$. Reactivity against GQ1b could be shown in monomeric IgG fractions pre- and post-IVIg treatment as well as to a higher extent in dimeric IgG fraction post-IVIg treatment (Fig. 2), suggesting that IgG dimers which occur after IVIg treatment in those two patients are formed by complexes that include autoantibodies and their presumed anti-idiotypes in IVIg preparations.

\section{Post-IVIg dimeric IgG fractions show immune reactivity against peripheral nerve fibers}

Based on our experiments using GQ1b and AchR antibody-positive sera, we further anticipated that IgG dimers that occur after IVIg treatment in CIDP patients may also contain autoantibodies and their IVIg-derived anti-idiotypes. We therefore used post-IVIg treatmentderived IgG dimers for screening for autoimmune reactivity against neuronal or Schwann cell epitopes in peripheral nerve fibers. Four out of ten tested dimeric IgG samples stained axons and/or myelin of peripheral nerve fibers (Fig. 3).

This immunofluorescence was not detected in monomeric or dimeric IVIg fractions, pre-dimeric IgG, or from post-dimeric IgG derived from patients with MFS or MG. Pre-treatment of tissue samples with methanol/

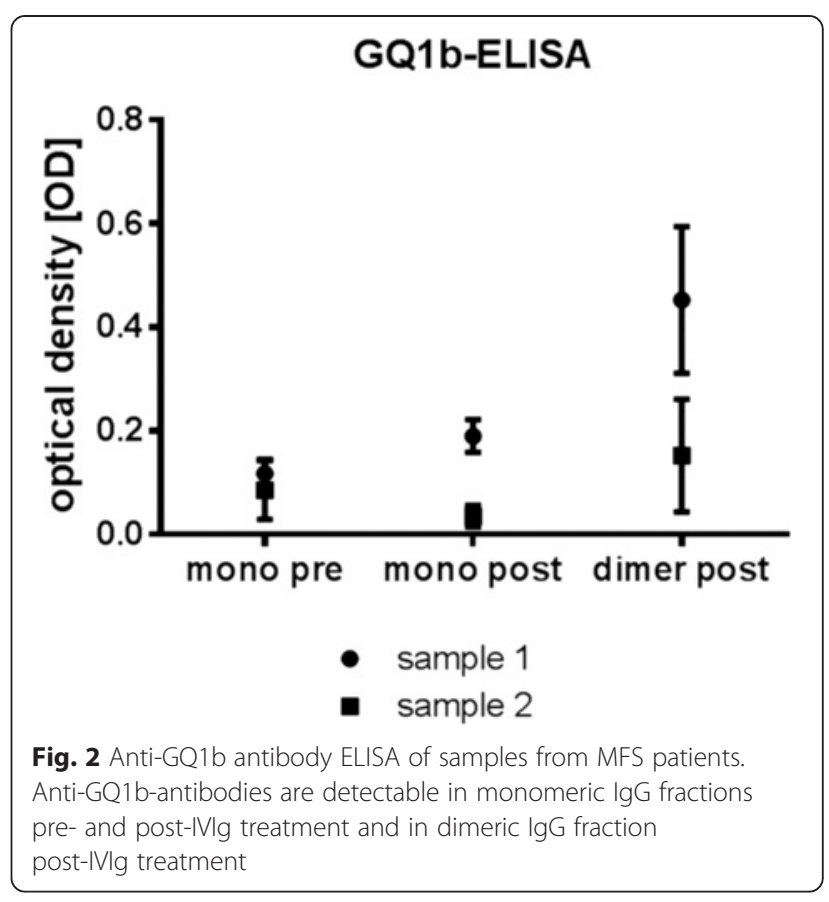

chloroform abrogated staining with dimeric IgG, indicating that those epitopes are membrane associated. From these data, we conclude that dimeric IgG contains antibodies that may recognize epitopes expressed on peripheral axons and Schwann cells.

\section{Discussion}

Our study is the first that characterizes the nature of dimeric IgG fractions that emerge during treatment with IVIg in an immune-mediated neurological condition. We demonstrate here that IVIg treatment induces the formation of variable amounts of IgG dimers in CIDP patients. The amount of newly formed IgG dimers in vivo is associated with clinical stabilization and/or improvement during IVIg treatment.

Consistent with previous studies that reported only low levels of naturally occurring IgG dimers in healthy individuals $[21,24,25]$, we found that the overall amount of IgG dimers in serum from controls and CIDP patients was considerably small. In contrast, commercial IVIg preparations contained higher amounts of IgG dimers. It is well known that pooled IgG from different donors contains usually higher amounts of IgG dimers; the amount of IgG dimers in commercial IVIg preparations varies between 5 and $15 \%$, depending on age, storage, formulation, and presence of chemical stabilizers $[18,19,26]$.

The intravenous application of polyclonal IgG (IVIg) resulted in a marked and rapid increase of IgG dimer serum levels after treatment in most of our patients. Those levels differed and were mostly higher as one 


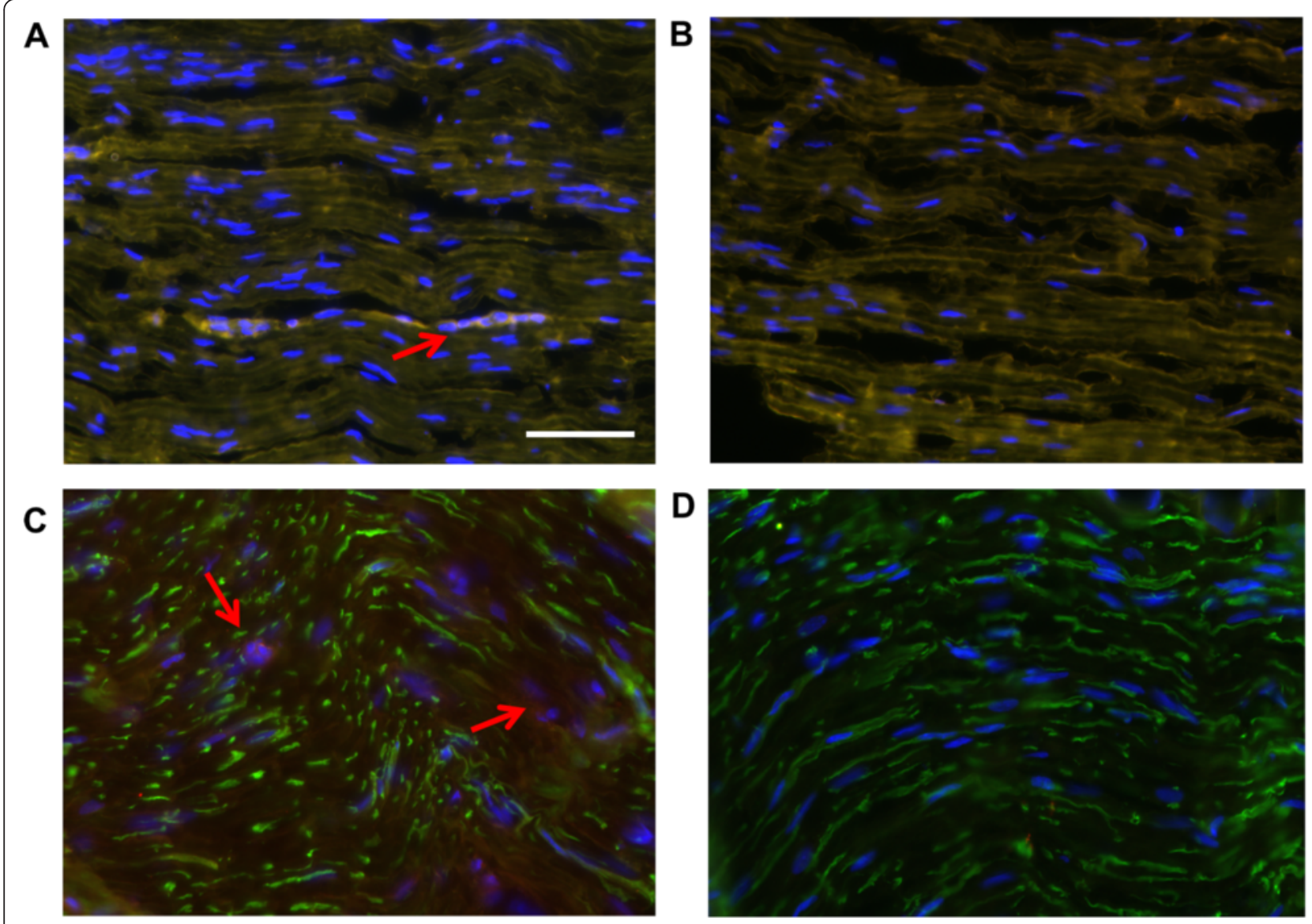

Fig. 3 Monomerized dimeric lgG fractions of CIDP patients stained axonal and myelin structures of the peripheral nerve. a Double immunofluorescence labeling with Hoechst counterstain (cell nuclei, blue) revealed binding of monomerized dimeric lgG fractions (red) from CIDP patients to myelin basic protein (green). b Control lgG showed no specific binding. Also, double immunofluorescence labeling with Hoechst counterstain (cell nuclei, blue) showed binding of monomerized dimeric lgG from CIDP patients (red) to axonal nerve structures (beta-III tubulin, green) (c), whereas no binding was observed in control staining (d) (scale bar $50 \mu \mathrm{m}$ )

would expect by simply adding the percentage of IgG dimers that are present in commercial IVIg preparations to the pre-existing IgG dimer amount. Therefore, it must be concluded that IVIg infusion results in the spontaneous formation of novel immune complexes in vivo.

The association of high IgG dimer levels with clinical stabilization in CIDP can be explained by interaction and subsequent neutralization of autoantibodies with anti-idiotypes that are present in IVIg preparations. IVIg contains anti-Id against a broad range of autoantibodies including anti-phospholipid [14], anti-DNA [15], antithyreoglobulin [27], and anti-neutrophil cytoplasmic antigen antibodies [28]. Those anti-Id interact with variable regions of other antibody molecules to form Idanti-Id complexes, which are detectable in dimeric IgG fractions $[16,21]$. A direct proof that IgG dimers derived from CIDP patients contain Id-anti-Id complexes would be desirable but cannot be provided due to the lack of commonly occurring, disease-specific autoantibodies in
CIDP $[29,30]$. However, our studies with post-treatment samples from two patients with MFS and one patient with MG support this concept and provide evidence that IVIg contains also anti-Id against anti-GQ1b and antiAchR antibodies, respectively. It further argues against the possibility that the IgG dimers in post-IVIg treatment samples contain exclusively IgG aggregates from IVIg preparations.

Although some of the monomerized IgG showed immunoreactivity against epitopes expressed on Schwann cells and peripheral neurons, the overall number of available samples was yet too small to define phenotypespecific staining patterns. This is supported from recent serological studies that reported novel disease-specific antibodies only in very small proportions (8.6 and $4.2 \%$ ) of cohorts of 46 [31] and 119 [32] CIDP patients. However, our immunolabeling studies with re-monomerized IgG fractions provide proof of concept that Id-anti-Id complexes that occur after IVIg treatment could be used 
as a novel approach to screen samples from CIDP patients in order to identify disease- or subgroup-specific autoantibodies. This approach is quite different from previous, mostly unsuccessful antibody screens that used whole serum fractions from CIDP patients [29, 30, 33]. The fact that a considerable proportion of patients' IgG showed reactivity against axonal tissue or Schwann cells may indicate an increased sensitivity of the strategy to use dimer IgG fractions for antibody testing. However, further validation studies with a larger cohort of patients are necessary to independently confirm the usefulness of IgG dimer quantification as a putative biomarker for IVIg treatment in CIDP.

Limitations of our study are a considerably small number of patients, particularly the proportion of CIDP patients who worsened during IVIg treatment. In addition, the patients in our cohort were only followed over a time period of 6 months which restricts conclusions about the utility of IgG dimers to serve as a putative biomarker for CIDP. Future studies are also necessary to determine dimer formation in IVIg-treatable conditions that are not associated with autoantibodies, such as primary immunodeficiency.

Nevertheless, the results of our study support the notion that anti-Id antibodies contribute to the beneficial effect of IVIg treatment in CIDP. Furthermore, the quantification of IgG dimer levels could be a feasible approach to identify IVIg-responsive CIDP patients. Further assessment of pre- and post-treatment IgG dimer levels in larger patient cohorts is warranted to explore their utility so serve as a potential biomarker for treatment response to IVIg in CIDP patients.

\section{Competing interests}

The authors declare that they have no competing interests.

\section{Authors' contributions}

$\mathrm{CR}$ and IB performed the experiments and analyzed the data. $\mathrm{HCL}$ supervised the study and designed the experiments. $C R$, IB, and $\mathrm{HCL}$ wrote the manuscript. All authors read and approved the final manuscript.

\section{Acknowledgements}

This work was supported by a Baxter Bio Science Grant. We thank Claudia Drapatz and Stefan Müller, CMMC, for excellent technical assistance.

\section{Author details}

'Department of Neurology, University Hospital Cologne, Cologne, Germany. ${ }^{2}$ Center of Molecular Medicine Cologne (CMMC), University of Cologne, Cologne, Germany. ${ }^{3}$ Cognitive Neuroscience, Institute of Neuroscience and Medicine (INM-3), Research Centre Jülich, Jülich, Germany.

Received: 1 May 2015 Accepted: 9 July 2015

Published online: 14 August 2015

\section{References}

1. Nobile-Orazio E. Chronic inflammatory demyelinating polyradiculoneuropathy and variants: where we are and where we should go. J Peripher Nerv Syst. 2014;19:2-13.

2. Dalakas MC. Advances in the diagnosis, pathogenesis and treatment of CIDP. Nat Rev Neurol. 2011;7:507-17.
3. Vallat JM, Sommer C, Magy L. Chronic inflammatory demyelinating polyradiculoneuropathy: diagnostic and therapeutic challenges for a treatable condition. Lancet Neurol. 2010;9:402-12.

4. Köller H, Kieseier BC, Jander S, Hartung HP. Chronic inflammatory demyelinating neuropathy. N Engl J Med. 2005;352:1343-56.

5. Lehmann HC, Hughes RA, Hartung HP. Treatment of chronic inflammatory demyelinating polyradiculoneuropathy. Handb Clin Neurol. 2013;115:415-27.

6. Eftimov F, Winer JB, Vermeulen M, de Haan R, van Schaik IN. Intravenous immunoglobulin for chronic inflammatory demyelinating polyradiculoneuropathy. Cochrane Database Syst Rev. 2013;12, CD001797.

7. Hughes RA, Donofrio P, Bril V, Dalakas MC, Deng C, Hanna K, et al. Intravenous immune globulin (10\% caprylate-chromatography purified) for the treatment of chronic inflammatory demyelinating polyradiculoneuropathy (ICE study): a randomised placebo-controlled trial. Lancet Neurol. 2008;7:136-44.

8. Buttmann M, Kaveri S, Hartung HP. Polyclonal immunoglobulin G for autoimmune demyelinating nervous system disorders. Trends Pharmacol Sci. 2013;34:445-57.

9. Lehmann $\mathrm{HC}$, Hartung HP. Plasma exchange and intravenous immunoglobulins: mechanism of action in immune-mediated neuropathies. J Neuroimmunol. 2011;231:61-9.

10. Ritter $C$, Förster $D$, Albrecht $P$, Hartung HP, Kieseier BC, Lehmann HC. IVIG regulates BAFF expression in patients with chronic inflammatory demyelinating polyneuropathy (CIDP). J Neuroimmunol. 2014;274:225-9.

11. Shoenfeld Y, Rauova L, Gilburd B, Kvapil F, Goldberg I, Kopolovic J, et al. Efficacy of IVIG affinity-purified anti-double-stranded DNA anti-idiotypic antibodies in the treatment of an experimental murine model of systemic lupus erythematosus. Int Immunol. 2002;14:1303-11.

12. Mimouni D, Blank M, Payne AS, Anhalt GJ, Avivi C, Barshack I, et al. Efficacy of intravenous immunoglobulin (IVIG) affinity-purified anti-desmoglein anti-idiotypic antibodies in the treatment of an experimental model of pemphigus vulgaris. Clin Exp Immunol. 2010;162:543-9.

13. Zandman-Goddard G, Blank M, Shoenfeld Y. Intravenous immunoglobulins in systemic lupus erythematosus: from the bench to the bedside. Lupus. 2009;18:884-8.

14. Caccavo D, Vaccaro F, Ferri GM, Amoroso A, Bonomo L. Anti-idiotypes against antiphospholipid antibodies are present in normal polyspecific immunoglobulins for therapeutic use. J Autoimmun. 1994;7:537-48.

15. Vassilev TL, Bineva IL, Dietrich G, Kaveri SV, Kazatchkine MD. Variable region-connected, dimeric fraction of intravenous immunoglobulin enriched in natural autoantibodies. J Autoimmun. 1995;8:405-13.

16. Schaub A, von Gunten S, Vogel M, Wymann S, Rüegsegger M, Stadler BM, et al. Dimeric IVIG contains natural anti-Siglec-9 autoantibodies and their anti-idiotypes. Allergy. 2011;66:1030-7.

17. Schaub A, Wymann S, Heller M, Ghielmetti M, Beleznay Z, Stadler BM, et al. Self-reactivity in the dimeric intravenous immunoglobulin fraction. Ann N Y Acad Sci. 2007:1110:681-93.

18. Bolli R, Woodtli K, Bärtschi M, Höfferer L, Lerch P. L-Proline reduces lgG dimer content and enhances the stability of intravenous immunoglobulin (IVIG) solutions. Biologicals. 2010;38:150-7.

19. Teeling JL, Jansen-Hendriks T, Kuijpers TW, de Haas M, van de Winkel JG, Hack CE, et al. Therapeutic efficacy of intravenous immunoglobulin preparations depends on the immunoglobulin $\mathrm{G}$ dimers: studies in experimental immune thrombocytopenia. Blood. 2001;98:1095-9.

20. Morgan AC, Rossen RD, Twomey JJ. Naturally occurring circulating immune complexes: normal human serum contains idiotype-anti-idiotype complexes dissociable by certain IgG antiglobulins. J Immunol. 1979;122:1672-80

21. Roux KH, Tankersley DL. A view of the human idiotypic repertoire Electron microscopic and immunologic analyses of spontaneous idiotype-anti-idiotype dimers in pooled human IgG. J Immunol. 1990;144:1387-95.

22. Joint task force of the European Federation of Neurological Societies and the Peripheral Nerve Society. European Federation of Neurological Societies/Peripheral Nerve Society Guideline on management of chronic inflammatory demyelinating polyradiculoneuropathy: report of a joint task force of the European Federation of Neurological Societies and the Peripheral Nerve Society - first revision. J Peripher Nerv Syst. 2010;15:1-9. 
23. Hughes R, Bensa S, Willison H, Van den Bergh P, Comi G, Illa I, et al. Randomized controlled trial of intravenous immunoglobulin versus oral prednisolone in chronic inflammatory demyelinating polyradiculoneuropathy. Ann Neurol. 2001;50:195-201.

24. Tankersley DL. Dimer formation in immunoglobulin preparations and speculations on the mechanism of action of intravenous immune globulin in autoimmune diseases. Immunol Rev. 1994;139:159-72.

25. Tankersley DL, Preston MS, Finlayson JS. Immunoglobulin G dimer: an idiotype-anti-idiotype complex. Mol Immunol. 1988;25:41-8.

26. Bleeker WK, Teeling JL, Verhoeven AJ, Rigter GM, Agterberg J, Tool AT, et al. Vasoactive side effects of intravenous immunoglobulin preparations in a rat model and their treatment with recombinant platelet-activating factor acetylhydrolase. Blood. 2000;95:1856-61.

27. Dietrich $G$, Kazatchkine MD. Normal immunoglobulin $G(\lg G)$ for therapeutic use (intravenous lg) contain antiidiotypic specificities against an immunodominant, disease-associated, cross-reactive idiotype of human anti-thyroglobulin autoantibodies. J Clin Invest. 1990:85:620-5.

28. Rossi F, Jayne DR, Lockwood CM, Kazatchkine MD. Anti-idiotypes against anti-neutrophil cytoplasmic antigen autoantibodies in normal human polyspecific lgG for therapeutic use and in the remission sera of patients with systemic vasculitis. Clin Exp Immunol. 1991;83:298-303.

29. Sanvito L, Makowska A, Mahdi-Rogers M, Hadden R, Peakman M, Gregson N, et al. Humoral and cellular immune responses to myelin protein peptides in chronic inflammatory demyelinating polyradiculoneuropathy. J Neurol Neurosurg Psychiatry. 2009;80:333-8.

30. Melendez-Vasquez C, Redford J, Choudhary PP, Gray IA, Maitland P, Gregson $\mathrm{NA}$, et al. Immunological investigation of chronic inflammatory demyelinating polyradiculoneuropathy. J Neuroimmunol. 1997:73:124-34.

31. Querol L, Nogales-Gadea G, Rojas-Garcia R, Martinez-Hernandez E, DiazManera J, Suárez-Calvet X, et al. Antibodies to contactin-1 in chronic inflammatory demyelinating polyneuropathy. Ann Neurol. 2013;73:370-80.

32. Ng JK, Malotka J, Kawakami N, Derfuss T, Khademi M, Olsson T, et al. Neurofascin as a target for autoantibodies in peripheral neuropathies. Neurology. 2012;79:2241-8.

33. Nobile-Orazio E, Giannotta C, Briani C. Anti-ganglioside complex IgM antibodies in multifocal motor neuropathy and chronic immune-mediated neuropathies. J Neuroimmunol. 2010;219:119-22.

\section{Submit your next manuscript to BioMed Central and take full advantage of:}

- Convenient online submission

- Thorough peer review

- No space constraints or color figure charges

- Immediate publication on acceptance

- Inclusion in PubMed, CAS, Scopus and Google Scholar

- Research which is freely available for redistribution

Submit your manuscript at www.biomedcentral.com/submit 